\title{
Histone Methyltransferase G9a Regulates Expression of Nuclear Receptors and Cytochrome P450 Enzymes in HepaRG Cells at Basal Level and in Fatty Acid Induced Steatosis
}

\author{
(1) Parimal Pande, (1) Xiao-bo Zhong, and Warren W. Ku \\ Non-Clinical Drug Safety, Boehringer Ingelheim Pharmaceuticals, Ridgefield, Connecticut (P.P., W.W.K.) and Department of \\ Pharmaceutical Sciences, School of Pharmacy, University of Connecticut, Storrs, Connecticut (P.P., X.-b.Z.)
}

Received July 22, 2020; accepted September 11, 2020

\begin{abstract}
Obesity and nonalcoholic fatty liver disease (NAFLD) affect expression and function of cytochrome P450 genes (P450s). The increased expression of inflammatory cytokines is a major driver of the downregulation of P450 expression in NAFLD. Decrease in P450 expression could potentially lead to drug-drug interaction, inefficient pharmacological effect of a drug, or hepatotoxicity. An epigenetic modifier, histone 3 lysine 9 methyl transferase enzyme (G9a), known to increase histone 3 lysine 9 methylation, is downregulated in diet-induced obesity animal models. In a liver-specific G9a knockout animal model, expression of P450s was downregulated. Currently, the role of G9a in regulation of P450s in steatosis is unknown. Our hypothesis is that in steatosis G9a plays a role in downregulation of P450 expression. In this study, we used HepaRG cells to induce steatosis using a combination of free fatty acids oleic acid and palmitic acid. The G9a was knocked down and overexpressed using small interfering RNA and adenovirus mediated approaches, respectively. Knockdown and overexpression of G9a in the absence of steatosis decreased and increased expression of nuclear receptors constitutive androstane receptor (CAR), pregnane
\end{abstract}

$X$ receptor, small heterodimer partner, and CYP2B6, 2E1, 2C8, 2C9, and 3A4, respectively. In steatotic conditions, overexpression of G9a prevented fatty acid mediated decreased expression of CAR, CYP2C19, 2C8, 7A1, and 3A4. Our current study suggests that G9a might serve as a key regulator of P450 expression at both the basal level and in early steatotic conditions. Single nucleotide polymorphism of G9a leading to loss/gain of function could lead to the poor metabolizer or ultrarapid metabolizer phenotypes.

\section{SIGNIFICANCE STATEMENT}

The current study demonstrates that histone modification enzyme G9a is involved in the regulation of expression of nuclear receptors constitutive androstane receptor, pregnane $X$ receptor, and small heterodimer partner as well as drug-metabolizing cytochrome P450s (P450s) at basal conditions and in fatty acid induced cellular model of steatosis. Histone 3 lysine 9 methylation should be considered together with histone 3 lysine 4 and histone 3 lysine 27 methylation as the epigenetic mechanisms controlling gene expression of P450s.

\section{Introduction}

Various factors affect cytochrome P450 (P450) expression and function, such as single nucleotide polymorphisms (SNPs), epigenetic influence, gender, age, and disease conditions. In nonalcoholic fatty liver disease (NAFLD), the expression of P450 genes is downregulated (Zanger and Schwab, 2013). In obese individuals, the prevalence of NAFLD increases with increasing body mass index (Fabbrini et al., 2010). Animal models, such as diet-induced obesity, or genetic models, such as $d b / d b$ or $o b / o b$, have shown to develop NAFLD with and without high fat diet treatment, respectively. In these animals, the

This project was supported by the Department of Non-clinical Drug Safety at Boehringer Ingelheim Pharmaceuticals, Ridgefield, CT.

https://doi.org/10.1124/dmd.120.000195. expression and activity of P450 genes were observed to be downregulated (Yoshinari et al., 2006; Ghose et al., 2011). In steatosis (accumulation of triglycerides in liver), the $\mathrm{P} 450$ expression is moderately affected, whereas in a more severe inflammatory nonalcoholic steatohepatitis (NASH), the expression is strongly affected due to inflammation (Lake et al., 2011). The in vivo phenotype of steatosis, which includes accumulation of lipid droplets, increased intracellular triglyceride, and downregulation of $\mathrm{P} 450$ genes, can be replicated in vitro by treating HepaRG and Huh-7 cells with fatty acids (FAs) (Woolsey et al., 2015; Michaut et al., 2016).

Drug metabolizing $\mathrm{P} 450$ genes are transcriptionally regulated by the xenobiotic nuclear receptors (NRs) constitutive androstane receptor (CAR), pregnane $\mathrm{X}$ receptor (PXR), and aryl hydrocarbon receptor (AHR). Similarly, an important bile acid synthesis gene, CYP7A1, is regulated by the small heterodimer partner (SHP). CAR and PXR are

ABBREVIATIONS: ACTB, beta-actin protein; AdV, adenovirus; AHR, aryl hydrocarbon receptor; CAR, constitutive androstane receptor; Cl, confidence interval; FA, fatty acid; FGF21, fibroblast growth factor 21; G9a, histone 3 lysine 9 methyl transferase; GAPDH, glyceraldehyde 3phosphate dehydrogenase; GFP, green fluorescent protein; H3K4, histone 3 lysine 4; H3K4me3, H3K4 trimethylation; H3K9, histone 3 lysine 9; H3K27me3, histone 3 lysine 27 trimethylation; HNF1 $\alpha$, hepatocyte nuclear factor 1 alpha; HNF4 $\alpha$, hepatocyte nuclear factor 4 alpha; IPA, isopropyl acetal; NAFLD, nonalcoholic fatty liver disease; NASH, nonalcoholic steatohepatitis; NR, nuclear receptor; OA, oleic acid; ORO, oil red O; P450, cytochrome P450; PA, palmitic acid; RT-PCR, real time quantitative polymerase chain reaction; PPAR $\alpha$, peroxisome proliferator activated receptor alpha; PXR, pregnane $X$ receptor; $R X R \alpha$, retinoid $X$ receptor alpha; siG9A, siRNA specific to G9A; siNC, siRNA negative control; siRNA, small interfering RNA; SHP, small heterodimer partner; SNP, single nucleotide polymorphism; TBP, TATA-binding protein. 
expressed in the cytosol and translocated to the nucleus to regulate gene expression upon trans-activation through binding of xenobiotic ligands, such as phenobarbital (Mutoh et al., 2013) and rifampicin (Kliewer et al., 2002), respectively (Timsit and Negishi, 2007). CAR, PXR, and peroxisome proliferator activated receptor alpha $(\operatorname{PPAR} \alpha)$ form a heterodimeric complex with retinoid $\mathrm{X}$ receptor alpha $(\mathrm{RXR} \alpha)$, which binds to the response elements of the promoter region of a target gene to regulate gene transcription.

NRs epigenetically regulate transcription of P450 genes through alterations of histone modifications. The histone proteins $\mathrm{H} 2 \mathrm{~A}, \mathrm{H} 2 \mathrm{~B}$, $\mathrm{H} 3$, and $\mathrm{H} 4$ are post-translationally modified at $\mathrm{N}$-terminal lysine residues. The methylation of lysine residues is associated with either active or repressed chromatin. Rifampicin treatment increases histone 3 lysine $4(\mathrm{H} 3 \mathrm{~K} 4)$ trimethylation $(\mathrm{H} 3 \mathrm{~K} 4 \mathrm{me} 3)$ and $\mathrm{H} 3$ acetylation, whereas it decreases histone 3 lysine 27 trimethylation $(\mathrm{H} 3 \mathrm{~K} 27 \mathrm{me} 3)$ at the $C Y P 3 A 4$ promoter to regulate its mRNA expression through activation of PXR (Yan et al., 2017). Similarly, $C Y P 2 E 1$ and $C Y P 2 C 9$ genes are regulated by $\mathrm{H} 3$ acetylation and $\mathrm{H} 3 \mathrm{~K} 27 \mathrm{me} 3$ modification (Yang et al., 2010; Englert et al., 2015). Treatment with 1,4-bis[2-(3,5-dichloropyridyloxy)] benzene in neonatal mice led to permanent activation of CAR and increased H3K4 mono-, di- and trimethylation and decreased histone 3 lysine 9 (H3K9) trimethylation at Cyp2b10 locus (Chen et al., 2012). The pregnenolone $16 \alpha$-carbonitrile treatment in neonatal mice caused persistent increase in expression of CYP3A11 due to increased $\mathrm{H} 3 \mathrm{~K} 4 \mathrm{me} 3$ and decreased $\mathrm{H} 3 \mathrm{~K} 27 \mathrm{me} 3$ at the Cyp3all promoter (Wang et al., 2019). Bile acid activated FXR increases expression of lysine specific demethylase 1, which removes $\mathrm{H} 3 \mathrm{~K} 4$ methylation to repress CYP7A1 expression and prevents bile toxicity (Kim et al., 2015). Therefore, the status of histone modifications at different residues determines either activation or repression of expression of P450s by activated NRs.

H3K9 mono-, di-, or trimethylation is associated with either activated or repressed chromatin. Histone 3 lysine 9 methyl transferase G9a methylates $\mathrm{H} 3 \mathrm{~K} 9$ to mono- and dimethylation at $\mathrm{N}$-terminal tail (Hyun et al., 2017), whereas suppressor of variegation 3-9 homolog 1 methylates to H3K9 trimethylation to repress gene expression (Lehnertz et al., 2003). G9a is transcribed by the gene euchromatic histone lysine methyl transferase 2. The G9a protein plays a dual role of activation or repression in epigenetic regulation of a gene based on its binding partners (Bittencourt et al., 2012; Chaturvedi et al., 2012; Shankar et al., 2013). The liver specific knockout of euchromatic histone-lysine $\mathrm{N}$-methyltransferase 2 (Ehmt2) in mice decreased expression of CAR, PXR, AHR, and SHP along with decreased expression of CYP2E1 and CYP3A11 (Lu et al., 2019). G9a was observed to be coimmunoprecipitated with SHP to repress CYP7A1 expression upon bile acid treatment in HepG2 cells by increasing H3K9 methylation (Fang et al., 2007).

FAs are endogenous ligands of $\operatorname{PPAR} \alpha$. FAs activate transcription of fibroblast growth factor 21 (FGF21) in response to fasting (Ge et al., 2012; Matikainen et al., 2012) to regulate triglycerides. The transcription factor, nuclear factor interleukin 3, also known as E4BP4, interacts with G9a to increase $\mathrm{H} 3 \mathrm{~K} 9$ dimetylation at the $F G F 21$ promoter to repress FGF21 gene expression (Tong et al., 2013). Previously, it was observed that treatment of Huh-7 cells with recombinant FGF21 protein resulted in decreased nuclear transport of PXR, leading to decreased CYP3A4 transcription (Woolsey et al., 2016).

Currently, the role of G9a in regulation of NRs and drug metabolizing P450 genes in steatosis has not been studied. In this study, we evaluated the role of G9a in regulation of P450s in free FA-induced steatosis. Differentiated HepaRG cells were selected as a model to perform gain of function and loss of function experiments due to similar expression of NR, P450s, and G9a to primary human hepatocytes (Hart et al., 2010).
A small interfering RNA (siRNA) mediated approach was used to selectively knock down G9a, whereas an adenovirus approach was used to overexpress G9a protein in HepaRG cells.

\section{Materials and Methods}

Chemicals and Reagents. HepaRG cells were purchased from Biopredic International (Rennes, France). ADD710 growth medium supplement and ADD720 differentiation medium supplement were purchased from Lonza (Morristown, NJ). Collagen I coated 6/24-well plates, William's E medium, Opti-MEM medium, Glutamax, silencer select siRNAs, and TaqMan probes were purchased from Thermo Fisher Scientific (Carlsbad, CA). Collagen I coated flasks were purchased from Corning (Corning, NY). siRNA negative control (siNC) (4390843) and siRNA specific to G9A (siG9A) (s21469) were purchased from Thermo Fisher Scientific. Adenovirus (AdV)-green fluorescent protein (GFP) (000541A) and AdV-G9a (087726A) were obtained from Applied Biologic Materials (Richmond, BC, Canada) at the titer $1 \times 10^{6} \mathrm{pfu} / \mathrm{ml}$. Oleic acid (OA) and palmitic acid (PA) were obtained from Sigma-Aldrich (St. Louis, MO).

Cell Culture. HepaRG cells were cultured according to the manufacturer's protocol (Biopredic International, Inc.). HepaRG cells were seeded in a tissue culture plate of desired format. Cells were allowed to grow in a HepaRG growth medium (William's E medium $500 \mathrm{ml}+65.5 \mathrm{ml}$ ADD710 + $5 \mathrm{ml}$ Glutamax) for 2 weeks, followed in a medium with 1:1 mixture of HepaRG growth medium and HepaRG differentiation medium (William's E Medium $500 \mathrm{ml}+76.7 \mathrm{ml}$ ADD720 + $5 \mathrm{ml}$ Glutamax) for 1 week, further in a HepaRG differentiation medium for another week to allow cells to be differentiated. Cells were then used for treatment. During growth and differentiation processes, media were replaced every 2 to 3 days. The differentiation of HepaRG cells was morphologically confirmed for the presence of hepatocyte islands and formation of bile canaliculi (Mayati et al., 2018).

siRNA Transfection. siRNA transfection was performed according to the manufacturer's protocol (Thermo Fisher Scientific). siRNA was diluted to $10 \mu \mathrm{M}$ concentration using nuclease free water. To transfect two wells of a 24-well plate, $1 \mu l$ of diluted siRNA was mixed with $50 \mu l$ Opti-MEM medium and $3 \mu l$ of Lipofectamine RNAiMAX reagent in $50 \mu 1$ Opti-MEM medium. The mixture was incubated at room temperature before using for transfection. Cells were incubated for 4 days before further analysis.

Adenovirus Mediated Transduction. Adenovirus transfections was performed according to the manufacturer's protocol provided by Applied Biologic Materials. Differentiated HepaRG cells were treated with 1:100 dilution of ViralPlus Transduction Enhancer (G698) to increase infectivity. Viral particles were incubated with cells for 1 hour to attach the virus. Medium was aspirated after 1 hour and replaced with the HepaRG differentiated medium, and incubation was performed for 4 days before further analysis.

RNA Isolation and TaqMan Quantitative Polymerase Chain Reaction. Total RNAs were isolated from HepaRG cells using a TRIzol reagent according to the manufacturer's protocol (Thermo Fisher Scientific, Waltham, MA). RNA concentration was measured by a Nano Drop spectrophotometer (Nano Drop Technologies, Wilmington, DE) at $260 \mathrm{~nm}$. Real-time quantitative polymerase chain reaction (RT-PCR) analysis was performed on Quant Studio 12K Flex instrument using an Advanced Fast Master Mix in a fast mode with off shelf TaqMan probes (Thermo Fisher Scientific) for gene expression analysis of TATA-binding protein (TBP), glyceraldehyde 3-phosphate dehydrogenase (GAPDH), G9a, PXR, CAR, RXR $\alpha$, AHR, SHP, FXR, PPAR $\alpha$, hepatocyte nuclear factor 1 alpha $(\mathrm{HNF} 1 \alpha)$, hepatocyte nuclear factor 4 alpha $(\mathrm{HNF} 4 \alpha)$, FGF21, CYP1A2, 2B6, 2C8, 2C9, 2C19, 2E1, 3A4, and 7A1. Relative mRNA levels were determined by normalizing examined gene expression against mRNA levels of TBP and GAPDH using the $2^{-\Delta \Delta \mathrm{Ct}}$ method (Livak and Schmittgen, 2001).

Western Blot. Cell lysates were prepared from treated HepaRG cells grown in a six-well culture plate using a radioimmunoprecipitation assay buffer. Protein concentrations were determined using a Pierce Bicinchoninic Acid Protein Assay Kit (23227; Thermo Fisher). About $50 \mu \mathrm{g}$ protein was loaded on a MiniPROTEAN 4\%-20\% precast gel (456-8095; BioRad) and transferred to a nitrocellulose membrane (170-4158; BioRad). The membrane was blocked with nonfat dry milk (5\% in Tris buffered-saline plus Tween 20) for 1 hour. After blocking, membrane was incubated with a primary antibody against G9a protein (1:1000 dilution in 5\% nonfat dry milk), CYP3A4 (1:1000), and beta-actin protein 
(ACTB) $(1: 1000)$ overnight at $4^{\circ} \mathrm{C}$. On the following day, the blot was incubated with a secondary antibody anti-rabbit $\mathrm{IgG}$ conjugated with horseradish peroxidase (1:5000 in 5\% nonfat dry milk) for 45 minutes. The blot was washed three times in $1 \times$ Tris-buffered saline plus Tween 20 solution before adding clarity Western chemiluminescence solution. The blot was imaged using BioRad ChemiDoc. Total protein normalization was performed with a stain-free total protein method using Image Laboratory 6.0 software.

CYP3A4 Activity Using P450-GLO Assay. Cells were washed with PBS before measuring CYP3A4 enzyme activity using a CYP3A4-GLO Assay (V9001; Promega). Three micromolar luciferin-isopropyl acetal (IPA) stock was prepared in HepaRG differentiation medium. Cells were treated with $50 \mu 1$ of $3 \mu \mathrm{M}$ luciferin-IPA for 1 hour at $37^{\circ} \mathrm{C}$. After completion of incubation, $25 \mu \mathrm{l}$ medium was aspirated and added to a white opaque plate. To each sample, $25 \mu \mathrm{l}$ CYP3A4/luciferin-IPA detection reagent was added, and the plate was incubated at room temperature for 20 minutes before measuring luminescence on a plate reader.

Treatment of HepaRG Cells with Fatty Acids. OA and PA were dissolved in DMSO to prepare a $1 \mathrm{M}$ stock solution. FAs were combined together in a 2:1 ratio of oleic to palmitic acid and further diluted to $600 \mu \mathrm{M}$ in DMSO for treatment. The diluted $600 \mu \mathrm{M}$ stock was diluted 100-fold in William's E medium supplemented with serum free induction medium supplement ( $100 \mathrm{ml}$ William's E $+1 \mathrm{ml}$ Glutamax $+2 \mathrm{ml} \mathrm{HepaRG}$ serum free induction medium supplement) for treatment of cells.

Oil Red O Staining. Cells were washed with PBS and fixed with $10 \%$ formalin (in PBS) for 30 minutes. Working oil red O (ORO) solution was prepared by mixing three parts of $0.05 \%$ ORO in isopropanol in two parts of water. Fixed cells were again washed with PBS, and working ORO was added for 20 minutes. The cells were washed a few times to remove excess ORO stain. Cells were counterstained with hematoxylin for 1 minute and washed with PBS three times. The cells were then viewed under a phase contrast microscope.

Cellular Viability Using CellTiter-GLO Assay (ATP Content). Cells were washed with PBS before measuring viability. Fifty microliters of HepaRG differentiation medium was mixed with $50 \mu \mathrm{l}$ of CellTiter-GLO (G7570; Promega) working solution. Cells were incubated for 10 minutes on a plate shaker with a moderate shaking speed. Luminescence was measured using a plate reader.

Lactate Dehydrogenase Release Assay. Fifty microliters of supernatant media was aspirated upon completion of FA treatment. Fifty microliters of CytoTox-ONE reagent was added to the aspirated media and incubated at room temperature for 10 minutes. The fluorescence was then measured using a plate reader.

Quantification of Intracellular Triglyceride. Intracellular triglyceride was quantified using the Triglyceride Quantification Kit (MAK266; Sigma) by following the manufacturer's protocol. A standard curve was generated by using serial dilution of a standard provided in the kit. Cells were lysed and treated with lipase to convert triglyceride to glycerol and FA. Glycerol is enzymatically oxidized to produce a fluorescence product. The fluorescence was measured using a plate reader.

Statistical Analysis. The data are presented as means \pm S.D. The significance of difference was determined using unpaired two-tailed Student's $t$ test. Each test was performed independently without corrections for multiple comparisons. Statistical analysis was performed using Prism 8 software. $P$ value less than 0.05 was deemed as statistically significant.

\section{Results}

Knockdown and Overexpression of G9a Alter Expression of Drug Metabolizing P450s in HepaRG Cells. To study the impact of G9a knockdown on P450 expression, HepaRG cells were treated with siG9a or siNC as a control. After 4 days of treatment, total RNAs were isolated from all samples ( $n=3$ to 4 per group), and gene expression analysis was performed using TaqMan probes specific for drug metabolizing P450 genes. The efficiency of G9a knockdown was evaluated at both mRNA and protein levels in the siRNA treated cells (Fig. 1A). G9a mRNA expression was decreased to $-1.8 \log _{2}$ fold change [95\% confidence interval $(\mathrm{CI}):-2.1$ to $-1.5 ; P=0.0005$ ] compared with the siNC control (Fig. 1A, left panel). At the protein
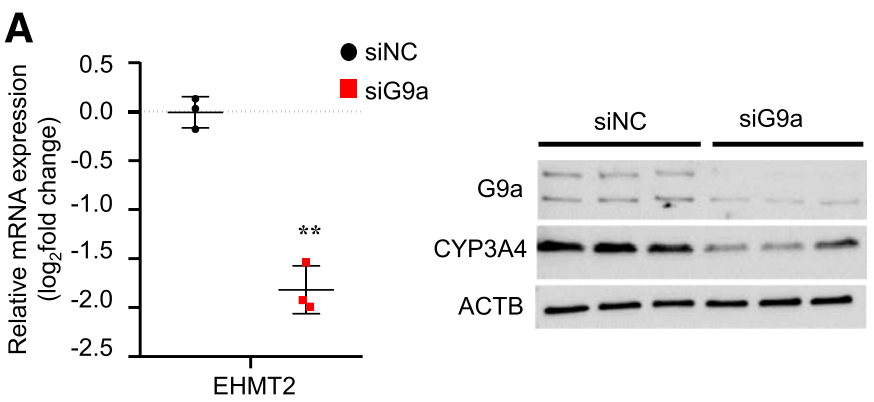

B
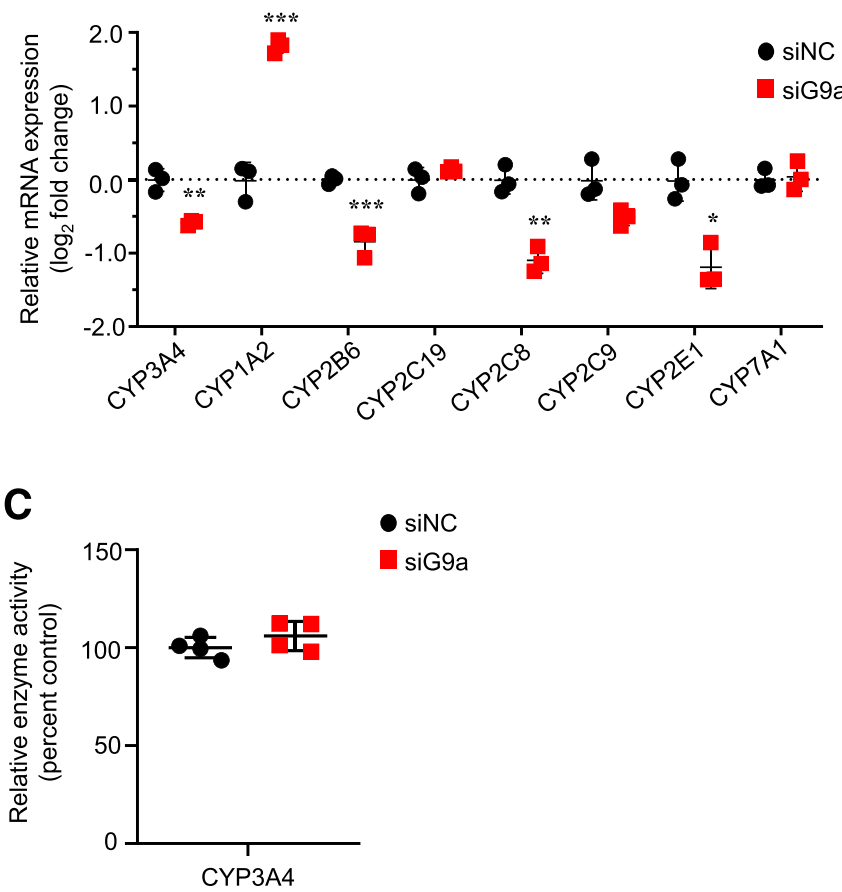

Fig. 1. Changes of gene expression by knockdown of G9a. (A) Efficiency of knockdown of G9a at mRNA (left) and protein (right) levels in HepaRG cells at day 4 after transfection with siG9a in comparison with siNC. CYP3A4 protein was also determined. ACTB was used as a housekeeping protein for a loading control. (B) Changes of mRNA expression of P450 genes at day 4 after siRNA treatment. RTPCR data analysis was performed using $2^{-\triangle \Delta C T}$ method. Mean cycle threshold values of TBP and GAPDH were used for $\triangle \mathrm{CT}$ calculations. (C) Enzyme activity of CYP3A4 at day 4 after siRNA treatment. Enzyme activity of CYP3A4 was determined by using the P450-Glo CYP3A4 assay. Fold changes of siG9a treated cells were calculated by comparing with the siNC control. The sample size for each treatment group is $n=3$ to 4 . Data are presented as means \pm S.D. from a single experiment. The experiment was repeated once to confirm the findings. $* P<0.05$; $* * P<0.01 ; * * * P<0.001$ compared with the controls using two-tailed unpaired Student's $t$ test.

level, the decrease in G9a expression was prominent on Western blot. Treatment of siRNA resulted in decreased expression of both isoforms of G9a protein (Fig. 1A, right panel). Similarly, the effect of G9a knockdown on mRNA expression of P450 genes was evaluated. The comparison of siG9a with siNC showed decreased mRNA expression of CYP3A4 $\left(-0.7 \log _{2}\right.$ fold change, $95 \% \mathrm{CI}:-0.8$ to $-0.5 ; P=$ $0.0054), 2 \mathrm{~B} 6(-0.8,95 \% \mathrm{CI}:-1.1$ to $-0.6 ; P=0.0007), 2 \mathrm{C} 8(-1.1$, $95 \% \mathrm{CI}:-1.3$ to $-0.9 ; P=0.0032)$, and $2 \mathrm{E} 1(-1.2,95 \% \mathrm{CI}:-1.6$ to $-0.9 ; P=0.011)$. In contrast, mRNA expression of CYP1A2 gene was increased (3.5 fold change, 95\% CI: 3.3-3.8; $P=0.0001$ ) compared with siNC control (Fig. 1B). The expression of CYP2C9, 2C19, and 7A1 mRNA did not change. The siRNA knockdown experiment showed that lower levels of G9a protein was associated 
with decreased expression of several P450s, including CYP2B6, 2C8, $2 \mathrm{E} 1$, and $3 \mathrm{~A} 4$, but increased expression of $1 \mathrm{~A} 2$. Interestingly, enzyme activity of CYP3A4 did not change in siG9a-treated cells in comparison with siNC treated cells (Fig. 1C), even though lower protein expression of CYP3A4 was observed in siG9a treated samples (Fig. 1A, right).

Furthermore, HepaRG cells were treated with adenovirus containing G9a coding domain sequence (AdV-G9a) in comparison with an adenovirus with green fluorescent protein (AdV-GFP) as a control to examine effects of G9a overexpression. Treatment with AdV-G9a caused a 244-fold (95\% CI: 240.6-248.9; $P=0.0001)$ increase in G9a mRNA expression compared with the AdV-GFP control (Fig. 2A, Left).

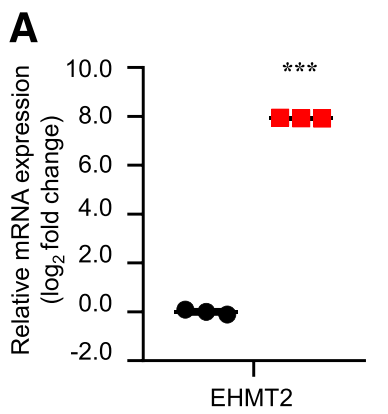

- AdV-GFP

- AdV-G9a

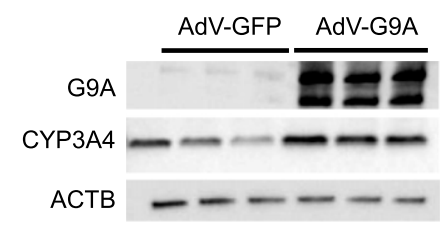

B
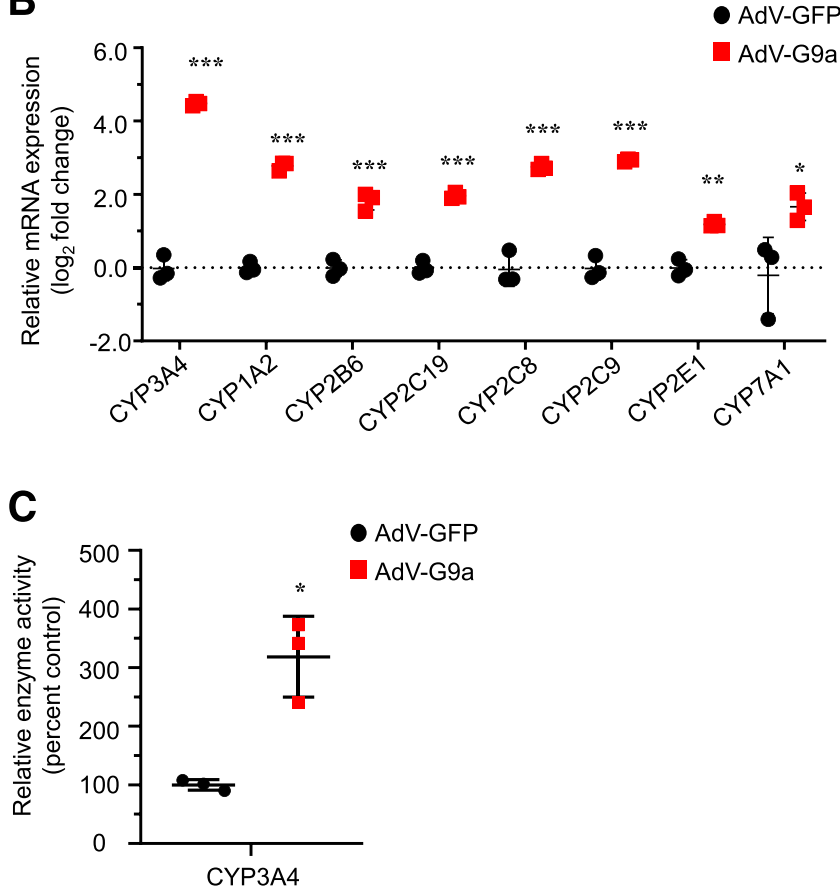

Fig. 2. Changes of gene expression by overexpression of G9a. (A) Efficiency of G9a overexpression at mRNA (left) and protein (right) levels in HepaRG cells at day 4 after transduction with AdV-G9a vs. AdV-GFP. CYP3A4 protein was also determined. ACTB was used as a housekeeping protein for a loading control. (B) Changes of expression of P450 genes at day 4 after adenovirus treatment. RT-PCR data analysis was performed using $2^{-\Delta \Delta C T}$ method. Mean cycle threshold values of TBP and GAPDH were used for $\Delta \mathrm{CT}$ calculations. (C) Enzyme activity of CYP3A4 at day 4 after adenovirus treatment. Enzyme activity of CYP3A4 was determined by using the P450-Glo CYP3A4 assay. Fold changes of AdV-G9a treated cells were calculated by comparing with the AdV-GFP control. The sample size for each treatment group is $n=3$. Data are presented as means \pm S.D. from a single experiment. The experiment was repeated once to confirm the findings. $* P<0.05$; $* * P<0.01 ; * * * P<0.001$ compared with the controls using two-tailed unpaired Student's $t$ test.
Similarly, an increase in G9a protein expression was also observed when assessed by Western blot (Fig. 2A, right). Compared with the AdV-GFP control, increased mRNA expression of CYP3A4 $(22.3$ fold, $95 \% \mathrm{CI}$ : 21.4-23.3; $P=0.0001), 1$ A2 (6.9 fold, 95\% CI: 6.3-7.5; $P=0.0001)$, 2B6 (3.6 fold, 95\% CI: 2.9-4.2, $P=0.0001$ ), 2C19 (3.9 fold, $95 \%$ CI: 3.6-4.2; $P=0.0001), 2 \mathrm{C} 8$ (6.7 fold, 95\% CI: 6.3-7.2; $P=0.0005), 2 \mathrm{C} 9$ (7.6 fold, 95\% CI: 7.4-7.9: $P=0.0001), 2$ E1 (2.3-fold, 95\% CI: $2.2-2.4$; $P=0.001)$, and 7A1 (1.7-fold, 95\% CI: $1.2-2.1 ; P=0.042)$ was observed (Fig. 2B). An increase in CYP3A4 enzyme activity to $318 \%$ (95\% CI: $240.8 \%-396.6 \% ; P=0.0297$ ) was also observed in the AdV-G9a treated cells in comparison with the AdV-GFP treated cells (Fig. 2C). Similarly, protein expression of CYP3A4 was increased in AdV-G9a treated samples (Fig. 2A, right).

Knockdown and Overexpression of G9a Alter Expression of Xenobiotic NRs in HepaRG Cells. Knockdown of G9a also resulted in alterations of mRNA expression of the NRs CAR, PXR, SHP, FXR, PPARa, and AHR, but not RXR $\alpha$ (Fig. 3A). Decreased mRNA expression of CAR $(-1.1$ fold, $95 \%$ CI: -1.3 to $-0.9 ; P=0.034)$, PXR ( -0.8 fold, $95 \% \mathrm{CI}:-0.8$ to $-0.7 ; P=0.0007)$, SHP $(-0.5$ fold, 95\% CI: -0.49 to $-0.56 ; P=0.0001)$, FXR $(-0.2$ fold, $95 \% \mathrm{CI}:-0.17$ to $-0.26 ; P=0.014), \operatorname{PPAR} \alpha(-0.4$ fold, $95 \% \mathrm{CI}:-0.28$ to $-0.59 ; P=$ 0.016) was observed upon knockdown of G9a compared with the siNC control. No change was observed in RXRa mRNA expression. The mRNA expression of AHR showed a 1.4-fold increase $(95 \% \mathrm{CI}$ : 1.2-1.6; $P=0.023$ ) (Fig. 3A). Overexpression of G9a showed the opposite effects of knockdown. In AdV-G9a overexpressing HepaRG cells, mRNA expression of CAR, PXR, and SHP was increased 7.7-fold (95\% CI: 7.6-7.8; $P=0.0004)$, twofold (95\% CI: 2.0-2.1; $P=0.0003$ ), and 1.5 -fold (95\% CI: $1.0-1.9 ; P=0.021$ ) respectively, compared with the AdV-GFP control (Fig. 3B). mRNA expression of $\operatorname{RXR} \alpha$, AHR, FXR, and PPAR $\alpha$ genes was not affected by G9a overexpression.

Free FA Treatment in HepaRG Cells as a Cellular Model of Steatosis. Differentiated HepaRG cells were treated with a combination of FAs for 24 hours, and DMSO treated samples were used as a control. ORO staining was performed to visualize intracellular accumulation of triglyceride. Accumulation of triglyceride was visible as early as 6 hours of treatment (data not shown) and more pronounced at 24 hours (Fig. 4A). This was accompanied by no changes in cell viability measured using Cell-Titer GLO assay (Fig. 4B) and lactate dehydrogenase release (Fig. 4C). Intracellular accumulation of triglyceride was measured using a fluorescence assay. A significant increase in accumulation of triglyceride was found in FA treated HepaRG cells at 24 hours (Fig. 4D). CYP3A4 mRNA expression was quantified using TaqMan and at the protein level by Western blot to examine the effects of FA treatment. CYP3A4 mRNA expression was decreased with -0.9 $\log _{2}$ fold change (95\% CI: -0.7 to $-1.1 ; P=0.017$ ) (Fig. $4 \mathrm{E}$ ) but with no change at the protein level (Fig. 4F).

G9a Modulation in Steatotic HepaRG Cells Alters the Expression of Drug Metabolizing P450s. Steatotic HepaRG cells were treated with siG9a or nonspecific siNC as a control to study the impact of G9a knockdown on P450 expression. In both siNC and siG9a treatment groups, FA treatment decreased P450 expression of CYP2C19, 2C8, 3A4, 2C9, 7A1, 1A2, and 2B6 compared with respective DMSO treated control groups (Fig. 5A).

Similarly, steatotic HepaRG cells were treated with AdV-G9a and AdV-GFP to evaluate effects on $\mathrm{P} 450$ expression. FA treatment resulted in decreased expression of CYP2C19, 2C8, 3A4, 2C9, 7A1, and 2B6 compared with AdV-GFP DMSO treated control (Fig. 5B). In AdV-G9a treatment groups, FA treatment did not decrease expression of CYP2C19, 2C8, and 3A4 as observed in siNC group treated with FA. CYP2C9 mRNA expression showed the same level of repression as seen in the siNC FA treated group. CYP7A1 expression was repressed to 


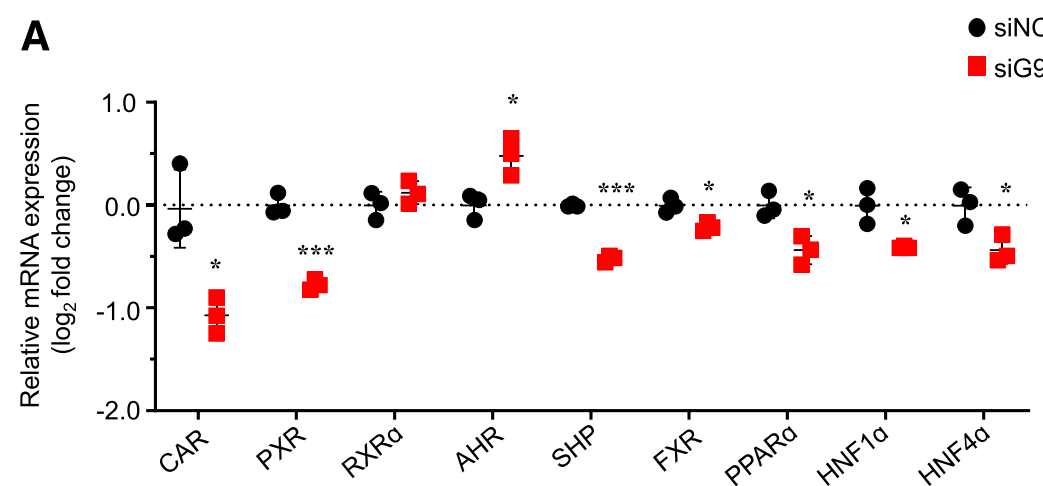

B

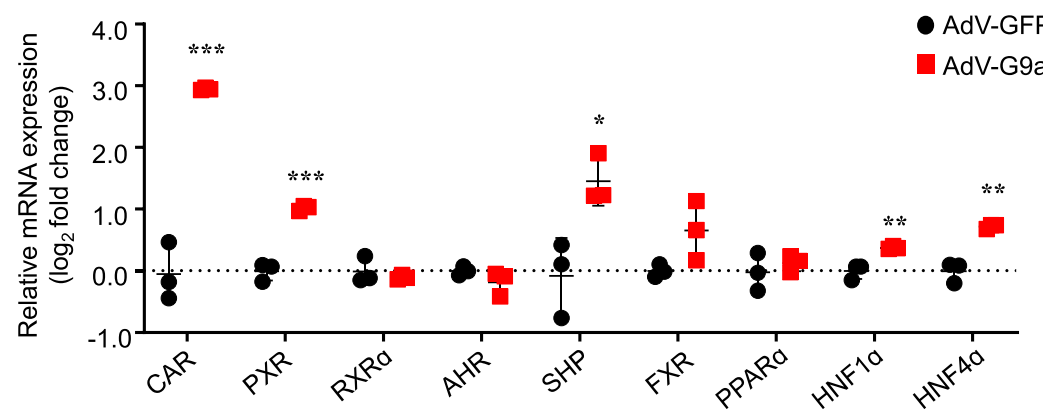

Fig. 3. Changes of mRNA expression of nuclear receptor genes by knockdown (A) and overexpression (B) of G9a. Expression of nuclear receptor genes at day 4 after siRNAs or adenovirus treatment was quantified by RT-PCR. The sample size for each treatment group is $n=3$. Data are presented as means \pm S.D. from a single experiment. The experiment was repeated once to confirm the findings. $* P<0.05 ; * * * P<0.001$ compared with the controls using two-tailed unpaired Student's $t$ test. a lesser extent than the siNC FA treated group. CYP1A2 and 2E1 expression were unaffected in both siNC and siG9a FA treated groups.

G9a Modulation in Steatotic HepaRG Cells Alters the Expression of NRs. The expression of NRs was measured after modulation of G9a expression in steatotic HepaRG cells. In the siNC group treated with FA, the expression of CAR, SHP, and PPAR $\alpha$ was decreased compared with siNC DMSO treated control group after 24 hours. The expression of PXR, AHR, and FXR showed slight to no change in expression (Fig. 6A). In the siG9a group treated with FA, expression of CAR and SHP was decreased to a greater extent than in the siNC FA treatment group. The expression of PXR, AHR, FXR, and PPAR $\alpha$ was not affected (Fig. 6B).

Similarly, in the AdV-GFP group treated with FA, the expression of CAR, SHP, and PPAR $\alpha$ was significantly downregulated compared with AdV-GFP DMSO treated controls. However, in the AdV-G9a group treated with FA, the expression of CAR was unaffected, whereas the decrease in expression of SHP and PPAR $\alpha$ was similar to that in the AdV-GFP FA treatment group. The expression of PXR, AHR, and FXR showed decreasing trend of expression in the siNC FA treatment group and comparable expression in the siG9a FA treatment group.

Alteration of mRNA Expression of FGF21 by Knockdown and Overexpression of G9a. Knockdown of G9a showed an increase of mRNA expression of FGF21 up to $2.1 \log _{2}$-fold (95\% CI: $1.8-2.3 ; P=$ 0.0073) in HepaRG cells (Fig. 7A), whereas overexpression of G9a resulted in decreased FGF21 mRNA expression to $2.8 \log _{2}$-fold (95\% CI: -3.0 to $-2.6 ; P=0.003$ ) (Fig. 7B). Similarly, treatment of FA followed by knockdown and overexpression of G9a showed increased FGF21 expression $2.4 \log _{2}$-fold $(95 \%$ CI: $2.0-2.8 ; P=$ $0.0005)$ and $3.1 \log _{2}$-fold (95\% CI: $\left.2.8-3.4 ; P=0.0001\right)$, respectively (Fig. 7, C and D).

Modulation of G9a Affects Hepatic Transcriptional Regulators at Basal Level but Not in a Steatosis Condition. Overexpression of G9a increased mRNA expression of both HNF1 $\alpha 1.3$ fold $(95 \% \mathrm{CI}$ : $1.27-1.32 ; P=0.007)$ and HNF4 $\alpha 1.65$ fold $(95 \% \mathrm{CI}: 1.6-1.7 ; P=$ 0.0019). Knockdown of G9a showed decreased expression of HNF1 $\alpha$
$-0.41 \log _{2}$-fold (95\% CI: -0.42 to $-0.39 ; P=0.016$ ) and HNF4 $\alpha$ $-0.43 \log _{2}$-fold (95\% CI: -0.59 to $-0.29 ; P=0.028$ ). Fatty acid treatment showed less than $0.5 \log _{2}$-fold expression increases of both $\mathrm{HNF} 1 \alpha$ and $\mathrm{HNF} 4 \alpha$ in knockdown and overexpression groups.

\section{Discussion}

Obesity and NAFLD affect expression and function of cytochrome P450 genes. Decreased P450 activity could potentially lead to drug-drug interaction, inefficient pharmacological effect of a drug, or hepatotoxicity. For example, decreased activity of CYP3A4 enzyme may lead to increased exposure of midazolam, a drug metabolized by CYP3A4 enzyme. Clinically, in a study when morbidly obese patients (body mass index 40-68) presumably having severe NAFLD were treated with midazolam orally and by intravenous route, the oral bioavailability was increased due to decreased intestinal and hepatic CYP3A4 enzyme function (Brill et al., 2015). In another study, the extent of increase in plasma concentration of midazolam was increased from simple steatosis to NASH. The increased concentration of plasma midazolam inversely correlated with hepatic mRNA expression of CYP3A4 gene in both simple steatosis and NASH when compared with healthy controls (Woolsey et al., 2015). Animal models of diet-induced obesity are used to study the regulation and function of P450s in NAFLD. In obese individuals with type 2 diabetes, higher levels of H3K9 dimethylation were found around interleukin-1A promoter and coding regions of the phosphatase and tensin homolog gene in monocytes (Miao et al., 2010). Similarly, H3 acetylation was observed to be increased at the promoter region of the tumor necrosis factor-alpha and cyclooxygenase-2 genes (Hou et al., 2011). Knockdown of lysine demethylase 3A in mice led to development of adult obesity and metabolic syndrome (Koza et al., 2006), suggesting that histone modification plays a key role in obesity. To study the regulation and function of P450s in humans, cellular models are employed to control the variations of in vivo studies. Various hepatocellular carcinoma cell lines, such as HepG2, Huh-7, and HepaRG cells, are preferred over primary human hepatocytes due to 
A

HepaRG DMSO 24hr

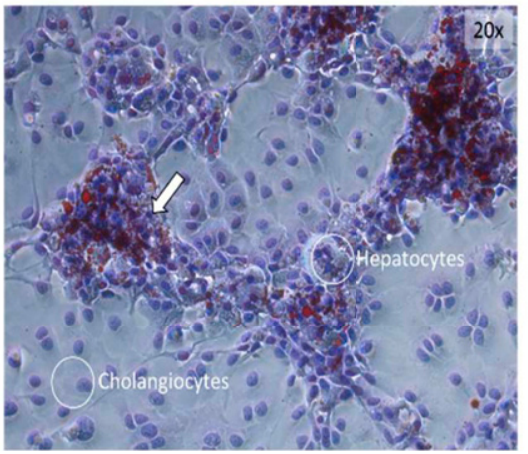

B
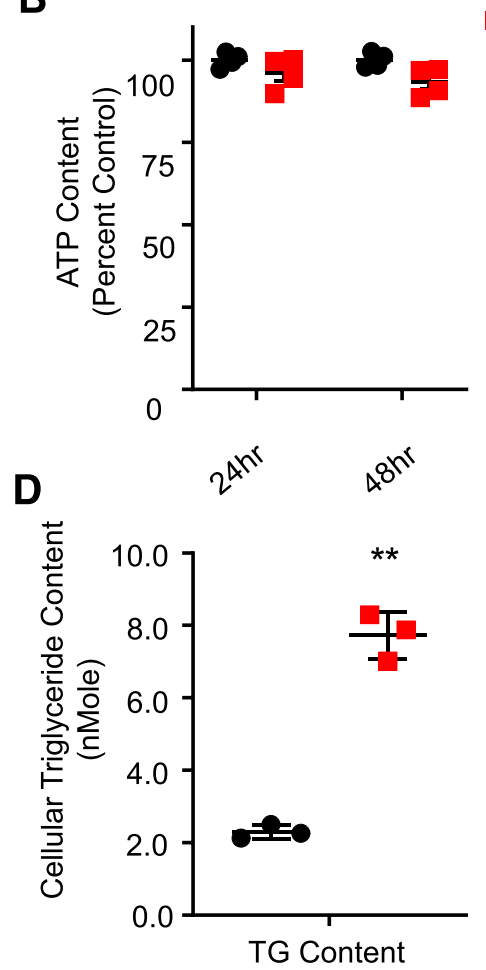

DMSO

OA/PA

$600 \mu \mathrm{M}$
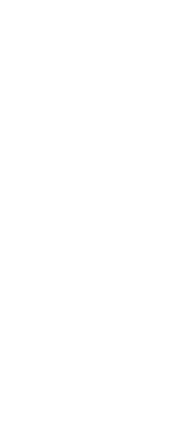

DMSO

$600 \mu \mathrm{M}$

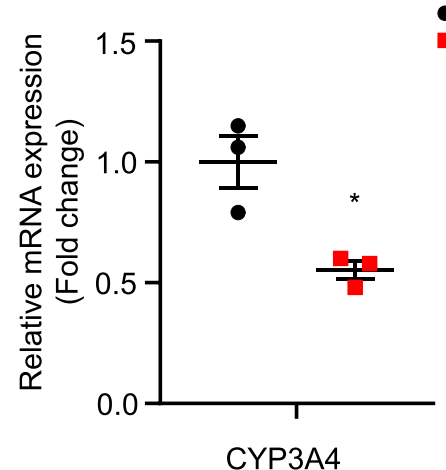

E

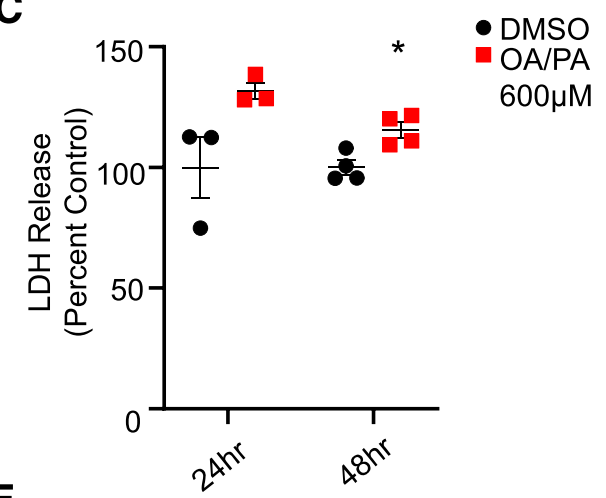

HepaRG OA/PA (2:1) $24 \mathrm{hr}$

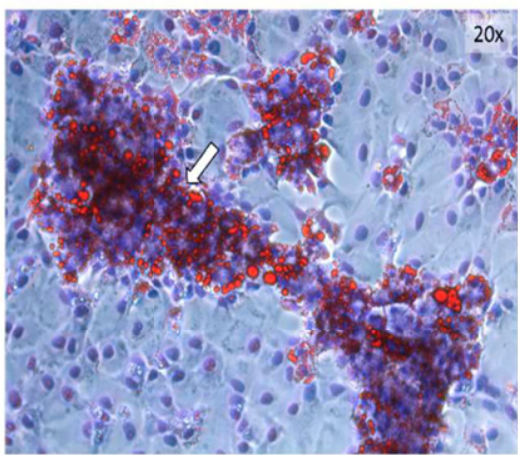

C

DMSO

OA/PA $600 \mu \mathrm{M}$

Fig. 4. (A) Accumulation of fatty acids in HepaRG cells visualized using Oil Red O staining 24 hours after treatment. (B) Cellular viability measured using quantification of ATP by CellTiter-GLO assay after 24 and 48 hours post-treatment. (C) Cellular damage caused by fatty acid treatment measured using lactate dehydrogenase release assay after 24 and 48 hours post-treatment. (D) Intracellular accumulation of triglycerides upon fatty acid treatment quantified using fluorescence assay at 24 hours post-treatment. (E) mRNA expression of CYP3A4 at 24 hours after fatty acid treatment measured using RT-PCR. (F) Protein expression of CYP3A4 using Western blot. Data analysis was performed using $2^{-\Delta \Delta \mathrm{CT}}$ method. Mean cycle threshold values of TBP and GAPDH were used for $\Delta C T$ calculations. The sample size for each treatment group is $n=3$ to 4 . Data are presented as means \pm S.D. from a single experiment. The experiment was repeated once to confirm the findings. $* P<0.05$; $* * P<0.01$ compared with the DMSO treated controls using two-tailed unpaired Student's $t$ test.

\section{$\mathbf{F}$}

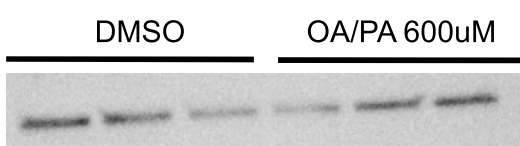

CYP3A4

ACTB

minimal experimental variability between batches of cells. HepaRG is a cell line of choice for majority of studies of drug metabolism and regulation due to similar expression and function of P450s and NRs (Hart et al., 2010).

In this study, G9a mediated regulation of P450s was studied in HepaRG cells. siRNA mediated knockdown and adenovirus mediated overexpression approaches were applied to study the transcriptional effect of G9a on P450s. In congruence with the liver specific G9a knockout experiment in mice, the knockdown of G9a led to decreased expression of majority of drug metabolizing P450s and their regulator NRs. To further strengthen the findings, the G9a overexpression showed increased NR and P450 expression. CYP3A4 was studied as a representative enzyme to investigate functional consequences. CYP3A4 is a major drug metabolizing enzyme, which metabolizes about $50 \%$ of marketed small molecule drugs (Shimada et al., 1994; Rendic, 2002). Knockdown of G9a did not affect CYP3A4 functional activity, whereas overexpression led to increased CYP3A4 enzyme activity.

In steatotic conditions, FA treatment increased intracellular accumulation of triglyceride. The mRNA expression of CYP3A4 was downregulated in HepaRG cells, replicating the in vivo steatotic phenotype. Knockdown of G9a and treatment of FA potentiated the decreased expression of CAR compared with FA treated siNC control, but no significant difference was observed in P450s and other NR expression. Conversely, overexpression of G9a prevented FA mediated repression of CAR and thus prevented downregulation of CYP3A4, 2C19, 2C8, and 7A1, suggesting CARdependent regulation of $\mathrm{P} 450$ s in steatosis is orchestrated by G9a. 

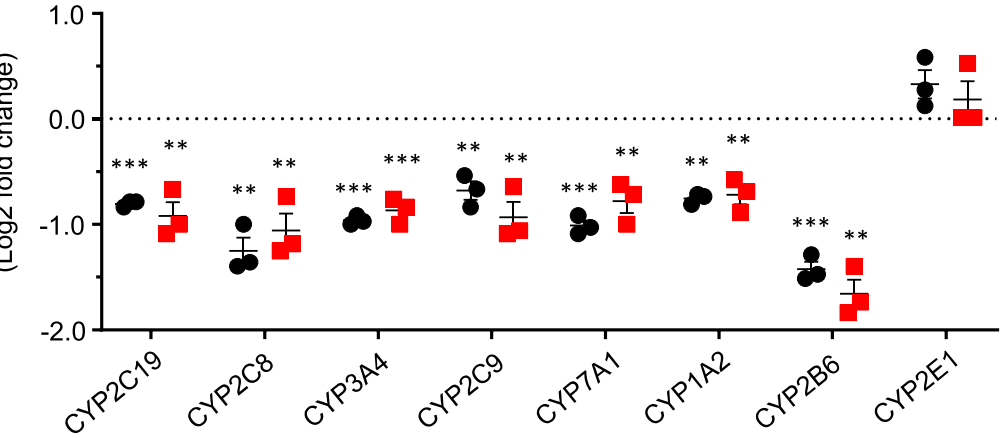

B

- AdV-GFP OA/PA

- AdV-G9a OA/PA

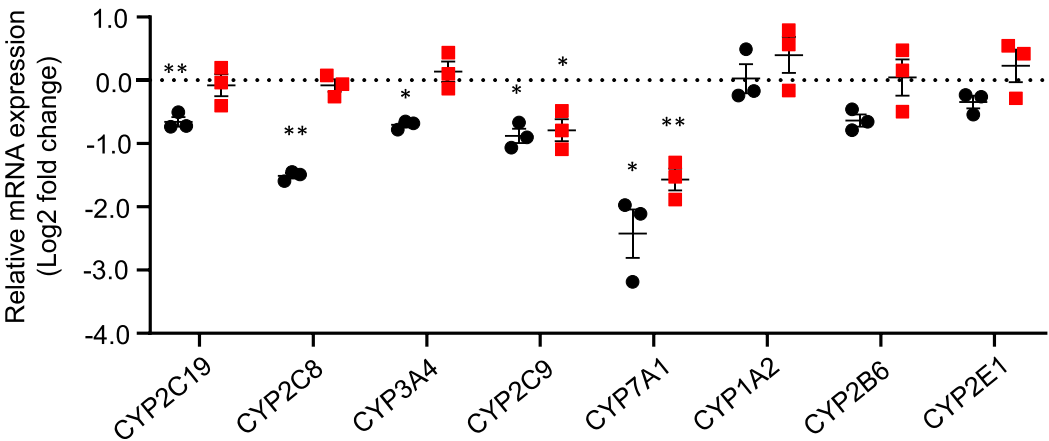

Fig. 5. Changes of mRNA expression of $\mathrm{P} 450$ genes after knockdown (A) and overexpression (B) of G9a followed by fatty acid treatment at 24 hours.RT-PCR data analysis was performed using $2^{-\Delta \Delta C T}$ method. Mean cycle threshold values of TBP and GAPDH were used for $\Delta \mathrm{CT}$ calculations. Fold changes of siNC OA/PA treated cells were calculated by comparing with the siNC DMSO control, siG9a OA/PA treated cells were calculated by comparing with siG9a DMSO control, AdV-GFP OA/PA treated cells were calculated by comparing with AdV-GFP DMSO control, and AdV-G9a OA/PA treated cells were calculated by comparing with AdV-G9a DMSO control. The sample size for each treatment group is $n=3$. Data are presented as means \pm S.D. from a single experiment. The experiment was repeated once to confirm the findings. $* P<0.05 ; * * P<0.01$; $* * * P<0.001$ compared with the controls using two-tailed unpaired Student's $t$ test.

Hepatic transcription factors $\mathrm{HNF} 1 \alpha$ and $\mathrm{HNF} 4 \alpha$, which are known to regulate a number of hepatic genes including P450s (Chen et al., 2018), showed an opposite effect upon G9a knockdown and overexpression, but no change was observed in the FA treatment condition. The data suggest that G9a-mediated modulation of hepatic transcription factors HNF $1 \alpha$ and $\mathrm{HNF} 4 \alpha$ could be responsible for CAR-mediated regulation of P450s at basal level. The adipokine FGF21, known to reduce plasma triglyceride levels by a variety of mechanisms (Schlein et al., 2016), was previously observed to regulate CYP3A4 expression by decreasing nuclear transport of PXR. FAs are endogenous ligands of
A

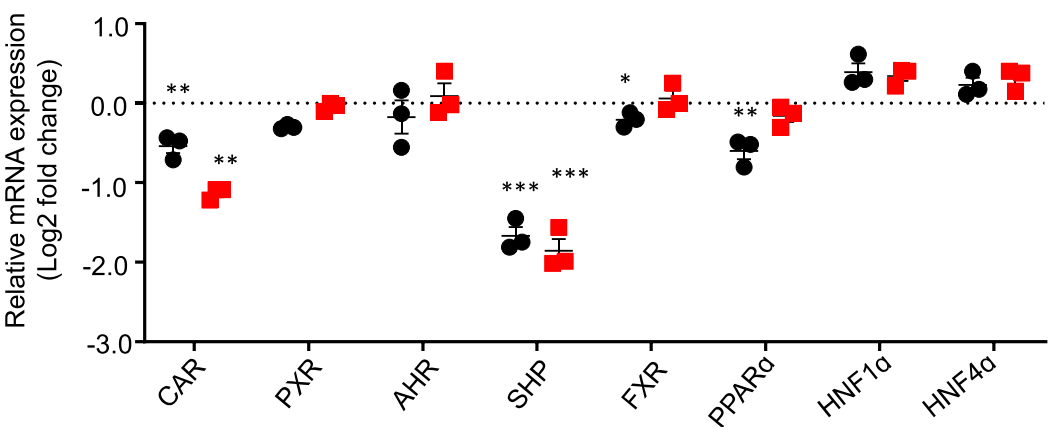

B

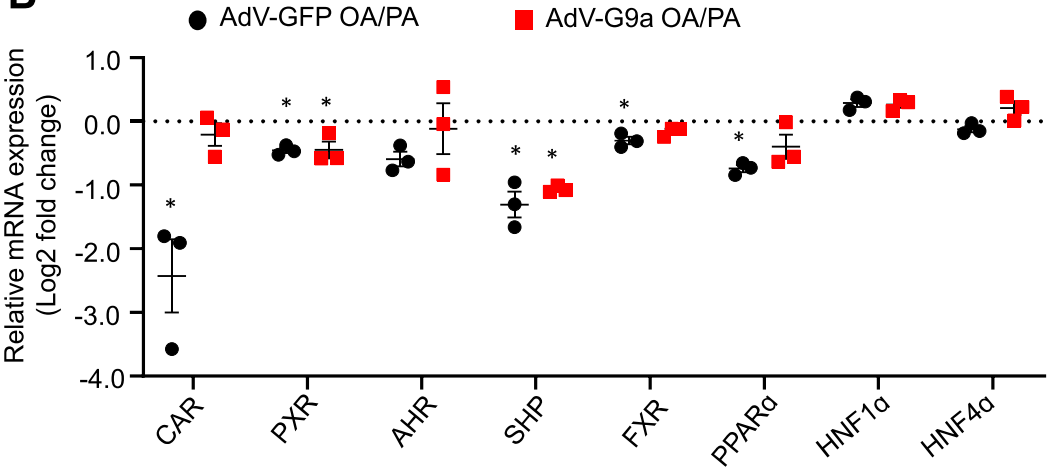

Fig. 6. Changes of mRNA expression of nuclear receptors after knockdown (A) and overexpression (B) of G9a followed by fatty acid treatment at 24 hours. RT-PCR data analysis was performed using $2^{-\Delta \Delta \mathrm{CT}}$ method. Mean cycle threshold values of TBP and GAPDH were used for $\triangle \mathrm{CT}$ calculations. Fold changes of siNC OA/PA treated cells were calculated by comparing with the siNC DMSO control, siG9a OA/PA treated cells were calculated by comparing with siG9a DMSO control, AdV-GFP OA/PA treated cells were calculated by comparing with AdV-GFP DMSO control, and AdV-G9a OA/PA treated cells were calculated by comparing with AdV-G9a DMSO control. The sample size for each treatment group is $n=3$. Data are presented as means \pm S.D. from a single experiment. The experiment was repeated once to confirm the findings. $* P<0.05$; ** $P<0.01 ; * * * P<0.001$ compared with the controls using two-tailed unpaired Student's $t$ test. 


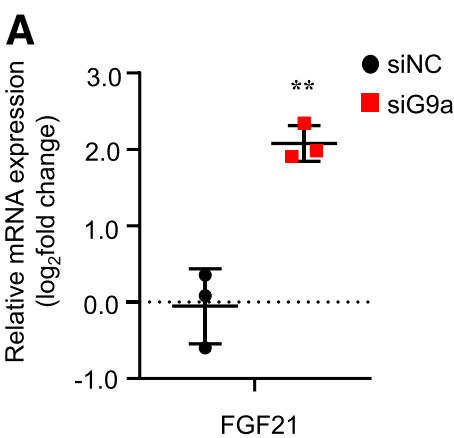

C

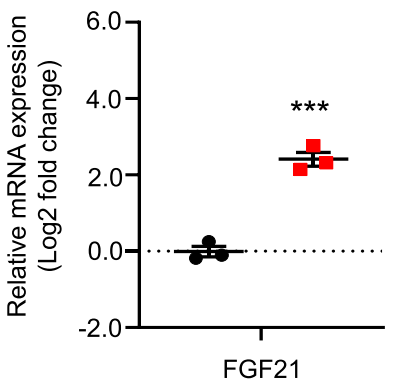

B

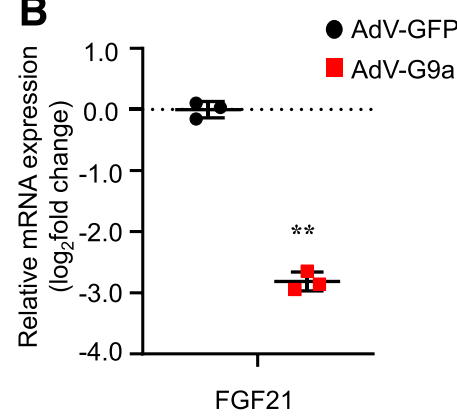

D

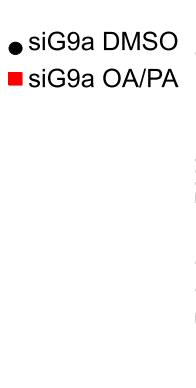

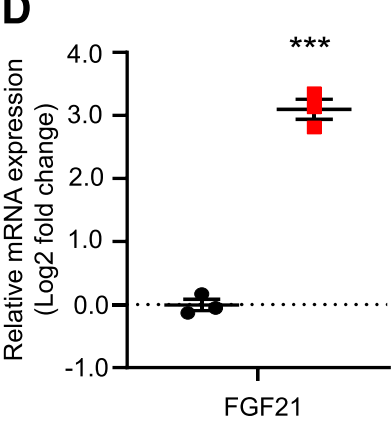

Fig. 7. Changes of mRNA expression of FGF21 gene by knockdown (A) and overexpression (B) of G9a at day 4. Changes of mRNA expression of nuclear receptors after knockdown (C) and overexpression (D) of G9a followed by fatty acid treatment at 24 hours. RT-PCR data analysis was performed using $2^{-\Delta \Delta C T}$ method. Mean cycle threshold values of TBP and GAPDH were used for $\Delta \mathrm{CT}$ calculations. The fold change of siG9a was calculated by comparing with siNC, AdV-G9a with AdV-GFP, siG9a OA/PA with siG9a DMSO, and AdV-G9a OA/PA with AdV-G9a DMSO. The sample size for each treatment group is $n=$ 3. Data are presented as means \pm S.D. from a single experiment. The experiment was repeated once to confirm the findings. $* * P<0.01 ; * * * P<0.001$ compared with the controls using two-tailed unpaired Student's $t$ test.
$\operatorname{PPAR} \alpha$, which activate transcription of FGF21 in response to fasting (Ge et al., 2012; Matikainen et al., 2012). In this study, FA treatment showed increased expression of FGF21 irrespective of G9a knockdown or overexpression. The expression of PPAR $\alpha$ was not affected by FA treatment. The functional activity of $\operatorname{PPAR} \alpha$ was speculated to be increased based on increased transcription of FGF21 mRNA. In the absence of steatosis, knockdown and overexpression of G9a showed increased and decreased expression of FGF21, respectively, suggesting an inhibitory effect of G9a on FGF21, as observed previously (Tong et al., 2013).

Xenobiotic ligands like 1,4-bis[2-(3,5-dichloropyridyloxy)] benzene bind directly to CAR to induce nuclear translocation, whereas ligands like phenobarbital increase nuclear translocation of CAR without direct binding to the ligand-binding domain (Yang and Wang, 2014). In many mammalian cells, CAR has shown a unique ability of constitutive activation in the absence of ligands (Choi et al., 1997; Dussault et al., 2002). Similarly, cyclin-dependent kinase signaling in mice increases nuclear translocation of PXR in the absence of ligand binding (Mackowiak and Wang, 2016), suggesting that besides ligand binding, various signaling pathways can increase nuclear translocation of CAR and PXR. Previously, it was observed that treatment of Huh-7 cells with recombinant FGF21 resulted in decreased nuclear transport of PXR and thus decreased CYP3A4 transcriptional expression (Woolsey et al., 2016). A direct relationship between CAR and FGF21 is currently not studied. In future experiments, the nuclear translocation of CAR could be assessed using immunofluorescence to understand the effect of G9a knockdown and overexpression on CAR mediated P450 regulation. Similarly, the effect of recombinant FGF21 on CAR nuclear translocation needs to be assessed because an inverse relationship of CAR and P450s was observed with FGF21 expression in this study.

In conclusion, the opposite effects produced by the knockdown and overexpression approaches suggest that G9a might be a key player in regulation of $\mathrm{P} 450$ genes through NRs. G9a is observed to positively regulate the basal expression of P450 genes in HepaRG cells. In a cellular model of steatosis, G9a was observed to regulate drug metabolizing P450s in a CAR-dependent manner. G9a is a histone methyl transferase enzyme responsible for the H3K9 mono- and dimethylation. In this study, we did not evaluate H3K9 methylation due to the scope of the study. In future experiments, the goal is to measure $\mathrm{H} 3 \mathrm{~K} 9$ methylation at the $C Y P 3 A 4, P X R$, and $C A R$ promoters to study the direct and indirect effect of G9a on CYP3A4 regulation. Transcriptional regulation is an interplay among a variety of histone modification changes. Increased expression of NR and P450s associated with G9a overexpression may have increased levels of H3K4me3 and $\mathrm{H} 3$ acetylation at the $C Y P 3 A 4$ promoter. Similarly, knockdown of G9a may have decreased levels of $\mathrm{H} 3 \mathrm{~K} 27 \mathrm{me} 3$ at the CYP3A4 promoter. Measuring other histone modifications along with $\mathrm{H} 3 \mathrm{~K} 9$ will highlight the mechanism of crosstalk between histone modifications and could be an objective of following studies. Similarly, the HepaRG cells were used as a representative cellular model to study the effect of G9a. The applicability of similar approaches of knockdown and overexpression in Huh-7 and primary human hepatocytes would provide more compelling evidence of G9a-mediated regulation of P450 expression. Clinically, there is limited information available about SNPs leading to G9a gain or loss of function. This study emphasizes a very important aspect of possible interindividual variability and drug-metabolism phenotype arising due to G9a SNPs. Undiagnosed steatosis or development of NAFLD in patients with G9a SNPs may misrepresent the P450 function, leading to hepatotoxicity or inefficient pharmacodynamic effect of drug treatment.

\section{Authorship Contributions}

Participated in research design: Pande, Zhong, Ku.

Conducted experiments: Pande.

Performed data analysis: Pande.

Wrote or contributed to the writing of the manuscript: Pande, Zhong, Ku.

\section{References}

Bittencourt D, Wu DY, Jeong KW, Gerke DS, Herviou L, Ianculescu I, Chodankar R, Siegmund KD, and Stallcup MR (2012) G9a functions as a molecular scaffold for assembly of transcriptional coactivators on a subset of glucocorticoid receptor target genes. Proc Natl Acad Sci USA 109:19673-19678.

Brill MJ, van Rongen A, van Dongen EP, van Ramshorst B, Hazebroek EJ, Darwich AS, RostamiHodjegan A, and Knibbe CA (2015) The pharmacokinetics of the CYP3A substrate midazolam in morbidly obese patients before and one year after bariatric surgery. Pharm Res 32:3927-3936. Chaturvedi CP, Somasundaram B, Singh K, Carpenedo RL, Stanford WL, Dilworth FJ, and Brand $\mathrm{M}$ (2012) Maintenance of gene silencing by the coordinate action of the H3K9 methyltransferase 
G9a/KMT1C and the H3K4 demethylase Jarid1a/KDM5A. Proc Natl Acad Sci USA 109: $18845-18850$.

Chen L, Bao Y, Piekos SC, Zhu K, Zhang L, and Zhong XB (2018) A transcriptional regulatory network containing nuclear receptors and long noncoding RNAs controls basal and drug-induced expression of cytochrome P450s in HepaRG cells. Mol Pharmacol 94:749-759.

Chen WD, Fu X, Dong B, Wang YD, Shiah S, Moore DD, and Huang W (2012) Neonata activation of the nuclear receptor CAR results in epigenetic memory and permanent change of drug metabolism in mouse liver. Hepatology 56:1499-1509.

Choi HS, Chung M, Tzameli I, Simha D, Lee YK, Seol W, and Moore DD (1997) Differential transactivation by two isoforms of the orphan nuclear hormone receptor CAR. J Biol Chem 272 23565-23571.

Dussault I, Lin M, Hollister K, Fan M, Termini J, Sherman MA, and Forman BM (2002) A structural model of the constitutive androstane receptor defines novel interactions that mediate ligand-independent activity. Mol Cell Biol 22:5270-5280.

Englert NA, Luo G, Goldstein JA, and Surapureddi S (2015) Epigenetic modification of histone 3 lysine 27: mediator subunit MED25 is required for the dissociation of polycomb repressive complex 2 from the promoter of cytochrome P450 2C9. J Biol Chem 290:2264-2278.

Fabbrini E, Sullivan S, and Klein S (2010) Obesity and nonalcoholic fatty liver disease: biochemical, metabolic, and clinical implications. Hepatology 51:679-689.

Fang S, Miao J, Xiang L, Ponugoti B, Treuter E, and Kemper JK (2007) Coordinated recruitment of histone methyltransferase G9a and other chromatin-modifying enzymes in SHP-mediated regulation of hepatic bile acid metabolism. Mol Cell Biol 27:1407-1424.

Ge X, Wang Y, Lam KSL, and Xu A (2012) Metabolic actions of FGF21: molecular mechanisms and therapeutic implications. Acta Pharm Sin B 2:350-357.

Ghose R, Omoluabi O, Gandhi A, Shah P, Strohacker K, Carpenter KC, McFarlin B, and Guo T (2011) Role of high-fat diet in regulation of gene expression of drug metabolizing enzymes and transporters. Life Sci 89:57-64.

Hart SN, Li Y, Nakamoto K, Subileau EA, Steen D, and Zhong XB (2010) A comparison of whole genome gene expression profiles of HepaRG cells and HepG2 cells to primary human hepatocytes and human liver tissues. Drug Metab Dispos 38:988-994.

Hou C, Zhao M, Li X, Li YJ, Lin Y, Lu QJ, and Zhou ZG (2011) [Histone H3 acetylation of tumor necrosis factor-alpha and cyclooxygenase-2 in patients with type 2 diabetes]. Zhonghua Yi Xue Za Zhi 91:1805-1808.

Hyun K, Jeon J, Park K, and Kim J (2017) Writing, erasing and reading histone lysine methylations. Exp Mol Med 49:e324.

Kim YC, Fang S, Byun S, Seok S, Kemper B, and Kemper JK (2015) Farnesoid X receptorinduced lysine-specific histone demethylase reduces hepatic bile acid levels and protects the liver against bile acid toxicity. Hepatology 62:220-231.

Kliewer SA, Goodwin B, and Willson TM (2002) The nuclear pregnane X receptor: a key regulator of xenobiotic metabolism. Endocr Rev 23:687-702.

Koza RA, Nikonova L, Hogan J, Rim JS, Mendoza T, Faulk C, Skaf J, and Kozak LP (2006) Changes in gene expression foreshadow diet-induced obesity in genetically identical mice. PLoS Genet 2: 81 .

Lake AD, Novak P, Fisher CD, Jackson JP, Hardwick RN, Billheimer DD, Klimecki WT, and Cherrington NJ (2011) Analysis of global and absorption, distribution, metabolism, and elimination gene expression in the progressive stages of human nonalcoholic fatty liver disease Drug Metab Dispos 39:1954-1960.

Lehnertz B, Ueda Y, Derijck AA, Braunschweig U, Perez-Burgos L, Kubicek S, Chen T, Li E, Jenuwein T, and Peters AH (2003) Suv39h-mediated histone H3 lysine 9 methylation directs DNA methylation to major satellite repeats at pericentric heterochromatin. Curr Biol $\mathbf{1 3}$ $1192-1200$.

Livak KJ and Schmittgen TD (2001) Analysis of relative gene expression data using real-time quantitative PCR and the 2(-Delta Delta C(T)) Method. Methods 25:402-408.

$\mathrm{Lu} \mathrm{H}$, Lei X, and Zhang Q (2019) Liver-specific knockout of histone methyltransferase G9a impairs liver maturation and dysregulates inflammatory, cytoprotective, and drug-processing genes. Xenobiotica 49:740-752.

Mackowiak B and Wang H (2016) Mechanisms of xenobiotic receptor activation: direct vs. in direct. Biochim Biophys Acta 1859:1130-1140.
Matikainen N, Taskinen MR, Stennabb S, Lundbom N, Hakkarainen A, Vaaralahti K, and Raivio T (2012) Decrease in circulating fibroblast growth factor 21 after an oral fat load is related to postprandial triglyceride-rich lipoproteins and liver fat. Eur J Endocrinol 166:487-492.

Mayati A, Moreau A, Le Vée M, Bruyère A, Jouan E, Denizot C, Parmentier Y, and Fardel O (2018) Functional polarization of human hepatoma HepaRG cells in response to forskolin. Sci Rep 8:16115.

Miao F, Wu X, Zhang L, Yaun Y-C, Riggs AD, and Natarajan R (2010) Genome-wide analysis of histone lysine methylation variations caused by diabetic conditions in human monocytes. $J$ Biol Chem 285: 14841

Michaut A, Le Guillou D, Moreau C, Bucher S, McGill MR, Martinais S, Gicquel T, Morel I, Robin MA, Jaeschke H, et al. (2016) A cellular model to study drug-induced liver injury in nonalcoholic fatty liver disease: application to acetaminophen. Toxicol Appl Pharmacol 292:40-55.

Mutoh S, Sobhany M, Moore R, Perera L, Pedersen L, Sueyoshi T, and Negishi M (2013) Phenobarbital indirectly activates the constitutive active androstane receptor (CAR) by inhibition of epidermal growth factor receptor signaling. Sci Signal 6:ra31.

Rendic S (2002) Summary of information on human CYP enzymes: human P450 metabolism data. Drug Metab Rev 34:83-448.

Schlein C, Talukdar S, Heine M, Fischer AW, Krott LM, Nilsson SK, Brenner MB, Heeren J, and Scheja L (2016) FGF21 lowers plasma triglycerides by accelerating lipoprotein catabolism in White and Brown adipose tissues. Cell Metab 23:441-453.

Shankar SR, Bahirvani AG, Rao VK, Bharathy N, Ow JR, and Taneja R (2013) G9a, a multipotent regulator of gene expression. Epigenetics 8:16-22.

Shimada T, Yamazaki H, Mimura M, Inui Y, and Guengerich FP (1994) Interindividual variations in human liver cytochrome P-450 enzymes involved in the oxidation of drugs, carcinogens and toxic chemicals: studies with liver microsomes of 30 Japanese and 30 Caucasians. J Pharmacol Exp Ther 270:414-423.

Timsit YE and Negishi M (2007) CAR and PXR: the xenobiotic-sensing receptors. Steroids 72 231-246.

Tong X, Zhang D, Buelow K, Guha A, Arthurs B, Brady HJ, and Yin L (2013) Recruitment of histone methyltransferase G9a mediates transcriptional repression of Fgf21 gene by E4BP4 protein. J Biol Chem 288:5417-5425.

Wang P, Liu G, Nie Y, Han S, Li J, Zhong XB, and Zhang L (2019) Epigenetic memory is involved in the persistent alterations of drug-processing genes in adult mice due to PCN-activated PXR during early life. Toxicol Sci DOI: 10.1093/toxsci/kfz177kfz177 [published ahead of print].

Woolsey SJ, Beaton MD, Mansell SE, Leon-Ponte M, Yu J, Pin CL, Adams PC, Kim RB, and Tirona RG (2016) A fibroblast growth factor 21-pregnane X receptor pathway downregulates hepatic CYP3A4 in nonalcoholic fatty liver disease. Mol Pharmacol 90:437-446.

Woolsey SJ, Mansell SE, Kim RB, Tirona RG, and Beaton MD (2015) CYP3A activity and expression in nonalcoholic fatty liver disease. Drug Metab Dispos 43:1484-1490.

Yan L, Wang Y, Liu J, Nie Y, Zhong XB, Kan Q, and Zhang L (2017) Alterations of histone modifications contribute to pregnane $\mathrm{X}$ receptor-mediated induction of CYP3A4 by rifampicin. Mol Pharmacol 92:113-123.

Yang H, Nie Y, Li Y, and Wan YJ (2010) Histone modification-mediated CYP2E1 gene expression and apoptosis of HepG2 cells. Exp Biol Med (Maywood) 235:32-39.

Yang H and Wang H (2014) Signaling control of the constitutive androstane receptor (CAR). Protein Cell 5:113-123.

Yoshinari K, Takagi S, Yoshimasa T, Sugatani J, and Miwa M (2006) Hepatic CYP3A expression is attenuated in obese mice fed a high-fat diet. Pharm Res 23:1188-1200.

Zanger UM and Schwab M (2013) Cytochrome P450 enzymes in drug metabolism: regulation of gene expression, enzyme activities, and impact of genetic variation. Pharmacol Ther 138 $103-141$.

Address correspondence to: Warren W. Ku, Non-Clinical Drug Safety, Boehringer Ingelheim Pharmaceuticals, 175 Briar Ridge Rd., Ridgefield, CT 06877. E-mail: warren.ku@boehringer-ingelheim.com 